\title{
Opacity Measurements: Extending the Range and Filling in the Gaps
}

C. A. Back, T. S. Perry, D. R. Bach, B. G. Wilson, C. A. Iglesias, O. L. Landen,

S. J. Davidson, and B. J. B. Crowley

This paper was prepared for submittal to the

Radiative Tranofer of Hot Dense Matter

Santa Barbara, California

November 4-8, 1996

March 17, 1997

This is a preprint of a paper intended for publication in a journdl or proceeding. Since changes my be made before publication, thio preprint is made available with the underntanding that it will not be cited or reproduced without the permitoion of the suther. 


\section{DISCLAIMER}

This document was prepared as an account of work sponsored by an agency of the United States Government. Neither the United States Government nor the University of California nor any of their employees, makes any warranty, express or implied, or assumes any legal liability or responsibility for the accuracy, completeness, or usefulness of any information, apparatus, product, or process disclosed, or represents that its use would not infringe privately owned rights. Reference herein to any specific commercial product, process, or service by trade name, trademark, mamufacturer, or otherwise, does not necessarily constitute or imply its endorsement, recommendation, or favoring by the United States Government or the University of California. The views and opinions of authors expressed herein do not necessarily state or reflect those of the United States Government or the University of California, and shall not be used for advertising or product endorsement purposes. 
Opacity Measurements: Extending the Range and Filling in the Gaps *

\author{
C. A. Back, T. S. Perry, D. R. Bach, B. G. Wilson, C. A. Igesias, O. L. Landen, \\ S. J. Davidson ', and B. J. B. Crowley'
}

Lawrence Livermore National Laboratory, L-473, P.O. Box 808

Livermore, CA 94551, USA

†Atomic Weapons Establishment, Aldermaston, Reading RG7 4PR, U. K.

A series of experiments to explore Ge opacity at temperatures where the M-shell is almost filled will be discussed. Data are obtained at lower temperatures than previously explored and allow us to investigate the role of atomic structure calculations and their impact on opacity scalings. The experiment uses the Nova laser to irradiate a gold hohlraum within which a $\mathrm{CH}$-tamped Ge sample is radiatively heated. A Nd backlight probes the sample 2 ns later to produce Ge spectral absorption features in the 1.2 - 1.5 $\mathrm{keV}$ energy range. Temperature is monitored by the use of an $\mathrm{Al}$ dopant and density is monitored by measuring the edge-on expansion of the sample. Temporal resolution of $\sim 200 \mathrm{ps}$ is obtained by using a short pulse backlight. Calculations in this photon energy region show significant changes in the spectral features.

\title{
I. Introduction
}

Point projection backlighting techniques have been successfully applied to obtain opacity measurements in the XUV and hard X-ray regimes for single temperature and density conditions of different $\mathrm{Z}$ elements. ${ }^{1}$ Obtaining these measurements has required careful design of the target and refinements of experimental techniques to obtain data with sufficient temporal and spatial resolution to permit comparisons with calculations. In these experiments we perform a systematic scan of the opacity as a function of temperature. In particular, we are targeting plasma conditions that give rise to ions with almost filled shells because calculations are not well benchmarked for these cases.

To measure opacity, one requires a single temperature and density in the sample. It is difficult to obtain several measurements during the same experiment as the sample is designed to equilibrate for a particular condition. Moreover, after the equilibration phase, there are gradients, so the measurements are compromised.

Design and target fabrication are crucial components and have become very sophisticated to attain the accuracy necessary for these type of measurements. 
This effort, however, has been very fruitful as previous experiments have demonstrated that experimental data can be used to benchmark calculations or applied to diagnose high energy density plasmas. For instance, meaurements of the opacity have been used to validate atomic physics models. ${ }^{2}, 3$ Measurements have also been used to measure the temperature of radiatively heated samples. 4 Yet another example is a more integrated measurement that experimentally measures the opacity in the regime of most importance to the Rosseland mean opacity. 5

The experimental technique of using absorption spectroscopy to measure the opacity of samples in local thermodynamic equilibrium (LTE) is the result of extensive efforts by several different laboratories over the past ten years. 1 Because laser-produced plasmas expand on timescales of ns, obtaining high quality data has depended on the development of diagnostics and techniques to calibrate, record, and analyze data acquired in sub-ns timescales. Temporal resolution has now become well established, either by short duration backlights or by gated detectors.

With these techniques in place we can now pursue a more global understanding of opacity. In the following we will discuss the methodology behind the research and also present experiments designed to access a particular regime of interest to the calculations.

\section{Systematic studies of the opacity}

The measurements of opacity on laser-produced plasmas have typically focused on open L-shell or open $\mathrm{M}$-shell regimes. For example, at a temperature of around $50 \mathrm{eV}$ and at a densities $\sim 1 \%$ of solid, aluminium has an open $2 \mathrm{p}$ shell which allows the study of $1 \mathrm{~s}-2 \mathrm{p}$ transitions, while germanium, under similar conditions, has an open $n=3$ shell, which allows the study of the $n=2-3$ transitions. In these half-filled shell cases, there are many different electron configurations, distributed among the valence and higher shells, and many possible transitions within each configuration. The spectrum will then exhibit a large number of individual lines having nearly identical transition energies so that adjacent lines overlap. This results in a spectrum and opacity that are insensitive to the details of the line profiles of the individual transitions. However, in other cases when the valence shell is on average nearly full or nearly empty, the number of available configurations is dramatically reduced resulting in both a simplification of the line spectrum in terms of the numbers of different lines present and, because individual lines tend to become more separated from their neighbors, an increasing sensitivity to the individual line profiles. This is particularly true at temperatures low enough to produce a negligible spectator population in higher electronic levels. Such temperature regimes are characteristic of these, and many other, laser-plasma experiments.

In the above argument, we use the word "configuration" in its most general sense where it refers to a general state of a many-electron system. Such general configurations are typically decomposed into the dominant central-field component and finer-scale term structure. The above argument applies equally to either type of structure. Term structure relates to the additional complexity 
due to non-central terms in the potential seen by each electron and thus simplifies as the system becomes more spherical, i.e. as shell become entirely full or empty.

The code CASSANDRA 6 has been used to study the systematics of LTE plasma spectra and this has been instrumental in guiding the experimental measurements. Calculations for $\mathrm{Ge}$ at densities $~ 1 \%$ of solid density show that the $\mathrm{M}$ shell becomes almost full as the temperatures fall below $\sim 20 \mathrm{eV}$ and almost empty for temperatures $\sim 200 \mathrm{eV}$. At each extreme, the opacity becomes increasingly sensitive to the details of the atomic physics model used in the calculation. Typically, the spectrum becomes more discrete because the number of transitions available to the bound electron configurations present in the plasma is reduced.

In the present experiments we study Ge plasmas at temperatures below 35 $\mathrm{eV}$, where the M-shell is nearly full and the plasma is less highly ionized. These plasmas are expected to exhibit increasingly discrete line spectra as the temperature decreases and the $3 \mathrm{~d}$ shell fills. Figure 1 shows a CASSANDRA calculation of the opacity of $\mathrm{Ge}$ at $12 \mathrm{eV}$ temperature and a density of $0.0531 \mathrm{~g} / \mathrm{cc}$. The contributions from the bound-free continuum and the boundbound lines are shown separately, and for this case, the spectrum is easily interpreted. The $2 \mathrm{p}$ and $2 \mathrm{~s}$ series limits occur around $1.3 \mathrm{keV}$ and $1.45 \mathrm{keV}$, respectively and account for the increases in continua opacity at these photon energies. The contributions from the $2 \mathrm{p}-3 \mathrm{~d}$ transitions at $1.2 \mathrm{keV}$, the $2 \mathrm{p}-4 \mathrm{~s}$ and $2 \mathrm{p}-4 \mathrm{~d}$ transitions at $1.3 \mathrm{keV}$, and the $2 \mathrm{~s}-4 \mathrm{p}$ transitions at $1.45 \mathrm{keV}$ are readily identifiable. At this temperature, only two ion stages are significantly present. Codes calculate that the line array widths become narrow as the $3 \mathrm{~d}$ shell fills.

Calculations reveal that the abrupt changes in detailed line structure as the temperature decreases from $50 \mathrm{eV}$ to $10 \mathrm{eV}$ is based on two factors. First, as the temperature decreases to $12 \mathrm{eV}$, fewer ions in the plasma will possess vacancies in the $\mathrm{M}$-shell and contribute to the oscillator strength of the $n=2-3$ lines. However, second, as the available number of configurations decreases, the spectrum becomes less complex, and the oscillator strength becomes distributed over fewer different line frequencies. These two factors have competing effects on the peak line opacity and whether the line heights increase or decrease over any given range of temperatures cannot readily be predicted. As an example of this, we can consider the effect of term structure alone. Both CASSANDRA and the STA code calculate the term structure in the UTA approximation, i.e. lines due to transitions between different terms in given central-field configurations are not treated in detail, but rather are treated in an approximation whereby all the different term-term transitions are represented by a homogeneous broadening of the line profile. Therefore as a shell fills and the term structure simplifies, the term broadening diminishes which results in an increase in the strength at the peak of the line.

Figure 2 shows the opacity calculated for different temperatures in a germanium plasma at a density of $0.0531 \mathrm{~g} / \mathrm{cc}$. At $50 \mathrm{eV}$, where previous experiments have been performed, the shell is approximately half full and features of the spectra are composites that can no longer be associated with 
particular transitions or even particular ions. Here the broadening and merging of line arrays due to complex multi-electron configurations is clearly evident. As the temperature decreases, fewer configurations are available to the electrons and fewer ions exist, thus the transition arrays are less broadened and the resulting spectrum is more discrete because there are fewer line overlaps. Calculations predict a sudden narrowing of the arrays as the final $3 \mathrm{~d}$ orbital fills resulting in a maximum opacity at the 2-4 line peaks. For germanium, this occurs at a temperature of $\sim 10 \mathrm{eV}$. From $50 \mathrm{eV}$ to $10 \mathrm{eV}$ the peak intensity increases by a factor of 2 .

Figure 3 shows the average level populations calculated for two temperatures in germanium. It is interesting and important to note that even for the $10 \mathrm{eV}$ case, the $3 \mathrm{~d}$ shell has no more than 1 or 2 vacancies left. The population distribution in the orbitals shows that partially filled orbitals can exist even in this case. By the time the temperature has reached $50 \mathrm{eV}$, none of the orbitals of the $n=3$ shell are full and in this case, the transition array widths are broadened.

In summary, the low temperature $12 \mathrm{eV}$ Ge case provides an example in which the $n=3$ shell is nearly full. The near closed $n=3$ principal shell results in a configurationally simpler plasma compared to the previous experiments which were performed at higher temperatures where the shell is approximately half filled. In half-filled shells, present for temperatures of $40-70 \mathrm{eV}$ for $\mathrm{Ge}$, the transition array widths depend only on the distribution of configurations in the plasma. In the regime of a filling shell, more spectral detailed features emerge and the spectrum becomes much more sensitive to the plasma conditions. We believe that experiments performed in such a regime provide critical tests of opacity modelling capability.

Because of the difficulties that codes, particularly those written with higher temperature applications in mind, can be expected to encounter when attempting to calculate this regime, we have sought to confirm the predictions of the CASSANDRA code by making direct comparisons between CASSANDRA and STA. ${ }^{7}$ For Ge, the predictions are quite similar. Both codes predict a similar break-up of the spectrum as the lines become more prominent. There are differences in the small scale structure and these can be attributed to differences in the way the codes model some aspects of the spectral features. For instance, the STA code is designed to resolve hierarchical spectral structure until it converges on the Rosseland mean opacity. Hence, it may not resolve finer detailed structure that exist in the experimental spectrum if these details do not affect the Rosseland mean value. Both codes treat term structure in the UTA approximation. However, while STA uses a gaussian array profile, CASSANDRA uses non-gaussian profile that distributes the calculated variance over a narrower central portion with broader wings. This will generally result in array components continuing to be resolved by CASSANDRA calculations, whereas the same arrays represented by gaussian profiles will have merged together in STA calculations. Experimental data to confirm the modelling of UTA profile shapes would be very helpful. 


\section{Experimental set-up}

Measurements of opacity in this $\mathrm{T}$ and $\rho$ regime are best done by absorption using point projection absorption spectroscopy because the ground state is in LTE. To achieve the necessary plasma conditions, the opacity samples are volumetrically heated by $x$-rays which radiatively heat the sample and eliminate laser-matter interactions that are difficult to quantify. Once the sample has expanded and equilibrated, a short duration backlight creates a burst of $x$-rays that probes the plasma.

The experimental set-up has been described by Perry, et al. 4 and a schematic of the target is shown in figure 5. Four beams of the Nova laser deliver $4.5-8.5 \mathrm{~kJ}$ of laser energy at $0.35 \mu \mathrm{m}$ in a $1 \mathrm{~ns}$ flat-topped pulse into an $8 \mathrm{~mm}$ long, $4 \mathrm{~mm}$ diameter hohlraum. The hohlraum is designed to create an $x$-ray flux having an effective blackbody temperature $\sim 30-40 \mathrm{eV}$ that radiatively heats the sample. Internal baffles to prevent laser light from directly irradiating the sample. Three other Nova beams are used at a wavelength of $0.53 \mu \mathrm{m}$ to provide a 200 ps pulse that creates the backlight source.

The sample is a thin deposit of $\mathrm{Ge}$ and $\mathrm{Al}$ that is suspended inside the hohlraum. It is a composed of very thin alternating layers of $\mathrm{Ge}$ and $\mathrm{Al}$ that are $\sim 50 \AA$ thick. Each experiment records the transmission of two samples of different thicknesses. This sample geometry allows the cross check of the opacity measurement. 8

The primary diagnostic is a Bragg crystal spectrometer that uses an RAP crystal bent on a $101.6 \mathrm{~mm}$ radius mount, providing a spectral resolution of $\sim 1.5$ $\mathrm{eV}$. The temporal resolution is determined by the duration of the $x$-ray backlight source which have been measured by time-resolved $x$-ray streak cameras to have a duration of $\sim 220 \mathrm{ps}$.

Figure 6 shows the transmission of the $\mathrm{Ge}$ and the $\mathrm{Al}$ sample. As the temperature decreases, the Ge spectra shifts to lower energies as expected, and the structure becomes more discrete. The plasma conditions are experimentally measured by the use of an $\mathrm{Al}$ "thermometer". The $\mathrm{Al}$ is deposited in thin layers that are interleaved with thin layers of $\mathrm{Ge}$ to create a multilayer target. Opacity measurements of $\mathrm{Al}$ at temperatures near $50 \mathrm{eV}$ have benchmarked this technique. At lower temperatures, this technique relies on the same type of ionization balance analysis to determine the temperature. For decreasing temperatures, the appearance of $n=3$ transitions can also be used to help determine the temperature. The experimental spectra for four different experiments is shown in figure 6. The F-like through C-like absorption features are the most prominent in these experiments. The codes $\mathrm{OPAL}^{9}$ and an MCDF/Saha codes both indicate that the data span a temperature range of 10 to $30 \mathrm{eV}$.

Another spectrometer and backlight, positioned orthogonally to that which measured the opacity, were used to obtain a radiograph of the sample. The edge-on expansion width is a measure of the density of the heated sample and is obtained at same time as the opacity measurement. The density was obtained by a fit to the absorption profile of the expanded foil. This measurement indicates 
that the density was $0.03 \mathrm{~g} / \mathrm{cc}$, which is less than expected. Fortunately, the opacity is much more sensitive to temperature. The higher density, therefore, does not significantly affect the structure of the spectra since it is not sufficient to create a significant change in the ionization balance.

A comparison of the data and calculations are shown in figure 7 with the opacity converted to transmission. The experimental transmission is a factor of two greater than the calculated values. The density measurement has the largest error bar, however, as mentioned above, an order of magnitude change in density yields changes in the transmission by less than $20 \%$. Therefore, the density is not sufficient to account for the discrepancy.

\section{Discussion and analysis}

Previous experiments have shown that the quality of the data depends on the stringent control of many experimental variables. Moreover, from previous experiences, we know there are two important aspects which may be responsible for the discrepancy. The first possibility is target fabrication. The samples were difficult to fabricate and contaminants have previously been found in similar samples. Since we have found impurities such as oxygen in similar opacity samples, we have investigated differing variations of a hypothetical contaminant to assess the impact that contaminants may have. The density measured during the deposition of the sample was $79.79 \mu \mathrm{g} / \mathrm{cm}^{2}$ of Ge and $48.59 \mu \mathrm{m} / \mathrm{cm}^{2}$ of $\mathrm{Al}$. If one assumes that through deposition error, the atomic percentage of the sample is actually $50 \%$ oxygen, the density would be effectively reduced to $70 \%$ of the nominal areal density. Unfortunately, this does not change the absoprtion enough to explain the discrepancy. In fact, we find that the contaminant would have to be as much as $80 \%$ by atomic weight if the discrepancy were due to this effect alone. However, this exceeds even generous estimates and is highly unlikely.

A second possible explanation is that the $\mathrm{CH}$ tamper is opaque. However the data is not consistent with this hypothesis. A $30 \mathrm{eV}$ blackbody radiation temperature will peak at photon energies of $\sim 90 \mathrm{eV}$ where the $\mathrm{CH}$ is still rather opaque. To volumetrically heat the sample, the mean free path of a photon at the peak of the black body should be $<1$. While this is a good rule of thumb, the expansion of the target must be taken into account. To ensure that the sample remains sufficiently dense and uniform the sample is tamped. Therefore higher optical depths can be tolerated as long as it becomes uniformly heated by the end of the $1 \mathrm{~ns}$ heating pulse. For these samples, the optical depth is $\sim 2.5$ through the $\mathrm{CH}$ and $\sim 5$ to the center of the sample. Experimentally we find the transmission of samples that were heated to approximately $30 \mathrm{eV}$ have a maximum transmission greater than that of cold $\mathrm{CH}$, which is evidence that it is not opaque. However, the colder sample at $20 \mathrm{eV}$ does have reduced transmission at photon energies $<1.2 \mathrm{keV}$ that may indicate that the sample is not sufficiently heated.

Unfortunately, neither of these possibilities can sufficiently account for the discrepancy of a factor of two in absorption. However, the cross check of the opacity deduced from the two foil thicknesses is not consistent and this can be 
indicative of two possible sources of error. First, this may indicate that the sample may have temperature gradients. At these low temperatures, the hydrodynamic expansion of the foil is very sensitive to the radiation temperature produced in the hohlraum. It is possible that the temperature is lower than expected and/or the $\mathrm{CH}$ tamper is partially opaque. In either case, temperature gradients may be present in the sample when the measurement is taken. Radiation temperatures near $20 \mathrm{eV}$ are difficult to measure by filtered $x$-ray diodes because of practical limitations. ${ }^{10}$ Radiation hydrodynamic simulations are expected to aid in understanding the gradients that may be present in this sample.

The last source of error may arise if the spectra is more discrete than calculated. If this is the case, the spectral resolution of the instrument would not sufficient to resolve these features. If the instrumental resolution is too low, then the net effect on the observed absorption would be to smear out the absorption features and the observed transmission will be larger than expected. The data show clear evidence that the spectra approaches the instrumental resolution and becomes very discrete at low temperatures. If this is the case, a higher resolution spectrometer will be needed for these measurements.

\section{Experimental plans}

Given the detailed structure expected in the opacity, the quality of the backlight is very important. The backlight used in these experiments was $\mathrm{Nd}$, which has structure in the $1.2-1.3 \mathrm{keV}$ range where these measurements are performed. Though analysis using $\mathrm{Nd}$ as a backlight can be performed, it is extremely arduous. Nd plasmas have a significant amount of structure due to the unresolved M-shell emission. Extreme care is necessary when analyzing the data to insure that the backlight structure does not introduce artificial structure into the data.

Recently, we have performed a series of experiments to improve the quality of the backlight for future experiments. To obtain a more spectrally smooth backlight, a Uranium was tested. The U emission is much smoother because 1.0 $1.3 \mathrm{keV}$ photon energies sample the continuum of the spectrum instead of the $M$ shell emission.

To increase signal level, we also experimented with using an angled fiber. The current backlight was created by two laser beams which irradiated either side of the foil. The source size of the backlight for a well aligned fiber is on the order of $40 \mu \mathrm{m}$. Fine tuning of the backlight angle is possible by the use of a rotational mount. When the backlight is edge-on, each beam theoretically contributes half the intensity. The angled geometry tests were performed at $5^{\circ}$ and $15^{\circ}$. These have shown that the intensity of the $U$ backlight can be greater than that of $\mathrm{Nd}$, moreover, because the emission is generated very close to the surface of the target, the use of one beam onto an angled backlight is equivalent to two beams on either side of it. Furthermore, it seems more efficient since the source will be more optically thick. The absolute intensity of the angled backlight emission and $\mathrm{Nd}$ emission are shown in figure 8 . The intensity does increase with the angle and the emission roughly follows a Lambertian 
dependence on angle. To obtain a backlight of source size that is comparable to that obtained with the $\mathrm{Nd}$ fiber, we plan to use an angle of $10^{\circ}$ for future experiments.

\section{Conclusions}

Techniques to study opacity in laser-produced plasmas are established and have advanced enough to allow a more systematic study of opacity. These are the first set of experiments to explore plasma conditions where codes have predicted a breakup of the spectral structure.

The experiments qualitatively agree with the calculations and show significant structure for $\mathrm{Ge}$ at 20 to $30 \mathrm{eV}$. However, the absolute values of the transmission are not in good agreement. Investigation of the experimental factors reveal that performing these measurements at lower temperatures may introduce some difficulties because of the hydrodynamics of the sample. This reemphasizes the importantance that these experiments be tailored to attain conditions of interest and maintain them for a sufficient time to perform the measurements.

Future measurements will scale to higher $Z$ elements so that the radiation temperature can be increased to a range in which it is more simple to perform the experiments. Finally, we will implement a higher resolution spectrometer to insure that the details of the spectral features are recorded.

\section{APPENDIX: Description of the CASSANDRA code}

CASSANDRA $^{6}$ is a second generation opacity code which uses the DiracHartree-Slater average atom model ${ }^{11}$ with Gunnarsson-Lundqvist exchange ${ }^{12}$ to calculate a self-consistent average-atom configuration for LTE plasma under specified conditions of temperature and density. Energies of actual atomic central-field configurations of more than 5 electrons are estimated by means of a Taylor series expansion, about the average atom, of the total energy as far as quadratic terms in the level populations, Ions having fewer than 5 bound electrons on average use an alternative procedure which applies a fit through discrete configurations. The required coefficients, which comprise the interaction matrix, are obtained by numerically differencing the Fock eigenvalues by reconverging the self-consistent field calculation onto configurations in the neighbourhood of the average atom. This procedure treats orbital relaxation which is the effect of allowing the one-body wavefunctions to vary in a manybody perturbative calculation. Because the Dirac equation is employed, the code uses $j$-coupling.

A generalised Ion Cell (GIC) model ${ }^{13}$ is used to determine the boundary conditions and the long range part of the average-atom potential. Plasma ion statistics are treated in the Grand Canonical Ensemble. The GIC model is equivalent to the ion-sphere model in the limit of strong coupling, but tends smoothly to Debye-Huckel under conditions of weak coupling. The total potential, which is that seen by a foreign test charge, is determined by matching the self-consistent atomic potential within a core region, whose radius is 
determined by self-consistency and continuity of the potential and charge distributions, to a screened Coulomb potential in the external (plasma) region.

Wavefunctions are calculated in the radial domain $(0, \infty)$, by numerical integration out to about $40 \%$ beyond the ion sphere radius to a maximum of 100 $\mathrm{au}$, and matched to an analytical representation beyond that. The model incorporates an implicit treatment of continuum lowering that, for a onecomponent plasma, is equivalent to the well known analytical formula fo Stewart and Pyatt ${ }^{14}$. The model however generalises to being able to treat two component plasmas (radially polarisable electrons) and mixtures of different elements with arbitrary degrees of mutual coupling.

It is recognised that this model only gives asymptotic level of the averaged continuum level. The discrete nature of neighbouring perturbers and fluctuations about the average are treated using a semiclassical model of line wings which considers the consequences of an upper state lying above the potential barrier between the ion and the nearest-neighbour perturber. The parameters of the wing profile come from the microfield distribution which uses a parametrisation of the nearest-neighbour perturber model. The merging of the line wings as a series approaches an edge gives rise to an apparent broadening of the edge.

Bound-bound transitions between atomic configurations in the $j j$-coupling representation are calculated in the dipole-approximation using average-atom wavefunctions with the exact form of the relatvistic dipole operator ${ }^{15}$. Line broadening includes Doppler broadening, quadratic Stark broadening, and linear Stark broadening for hydrogenic systems. UTA line profiles are represented as a sum of two gaussians whose overall variance is the sum of the variances due to each separate broadening process. The term structure contribution to the UTA width is estimated using Mozskowski's formula ${ }^{16}$. The bound-bound spectrum is calculated using a method of detailed configuration accounting in which valence shell electrons are treated in detail. Spectator electron configurations in higher-energy shells are treated statistically in terms of a "dielectronic satellite" 17 contribution to the line broadening.

Photoionisation (bound-free) transitions are computed in a similar manner for those upper states that can be supported on the numerical mesh. Use of an optimised semi-logarithmic mesh of the form

$$
x=\alpha r+\beta \ln (r)
$$

enables the numerical bound-free calculations to be extended to higher energies for a given size of mesh. At higher energies, the calculation is matched to a parametrisation based upon a universal fit to cold X-ray cross-section data.

Inverse-bremsstrahlung (free-free) is computed using a modified form of the Kramers formula that incorporates a treatment of low-frequency plasma collective effects (via the correction for plasma dispersion, see below) and 
collisional damping. Quantum mechanical effects, screening, degeneracy and relativistic effects are all treated via Gaunt factors. A parametrisation of electronelectron bremsstrahlung is also included.

The CASSANDRA absorption coefficient includes the correction for plasma dispersion via the refractive index ${ }^{18}$. Appropriate modifications are also made to the definitions of the integrated opacities (i.e., Rosseland or Planck mean).

Photon scattering includes detailed treatments of both incoherent (Compton) and coherent (Rayleigh) scattering, while taking account of electron degeneracy, thermal motion and induced scattering.

This work was performed under the auspices of the U.S.Department of Energy by Lawrence Livermore National Laboratory under contract no. W-7405-Eng-48. 


\section{FIGURES}

Figure 1. Spectrum of $\mathrm{Ge}$ at $12 \mathrm{eV}$. The features of the spectrum are identifiable because there are so few transitions possible.

Figure 2. Opacity of $\mathrm{Ge}$ at $\mathrm{T}=20,30,40,50 \mathrm{eV}$ calculated by the code Cassandra. As the temperature decreases, discrete transitions dominate the spectrum.

Figure 3. Fractional populations of Ge M-shell transitions calculated by the Cassandra code. Most experiments are performed at higher temperatures where the shells are half full. At lower temperatures where these data are taken, the $3 \mathrm{~d}$ shell has 1 or 2 vacancies.

Figure 4. Comparison of Ge M-shell opacity calculated by STA and Cassandra for two temperatures. Both codes predict a breakup in the structure at 10 to $20 \mathrm{eV}$ temperatures.

Figure 5. Photograph of the hohlraum target. The lasers enter into either end of the cylindrical hohlraum and create a radiation flux that heats the sample. The opacity sample is viewed through a diagnostic hole in the center where two samples are visible.

Figure 6 - Experimental data from a series of shots. The Ge opacity is measured in the $1.2-1.4 \mathrm{keV}$ range. The $\mathrm{Al}$ is used to obtain temperature measurements.

Figure 7. The data, represented by the black line, shows more discrete spectral features at low temperatures. This is qualitatively consistent with the calculations.

Figure 8. A comparison of different backlights shows that a $U$ backlight is preferable to a Nd backlight because it is spectrally smoother and more intense. The Uranium will be used on future experiments. 


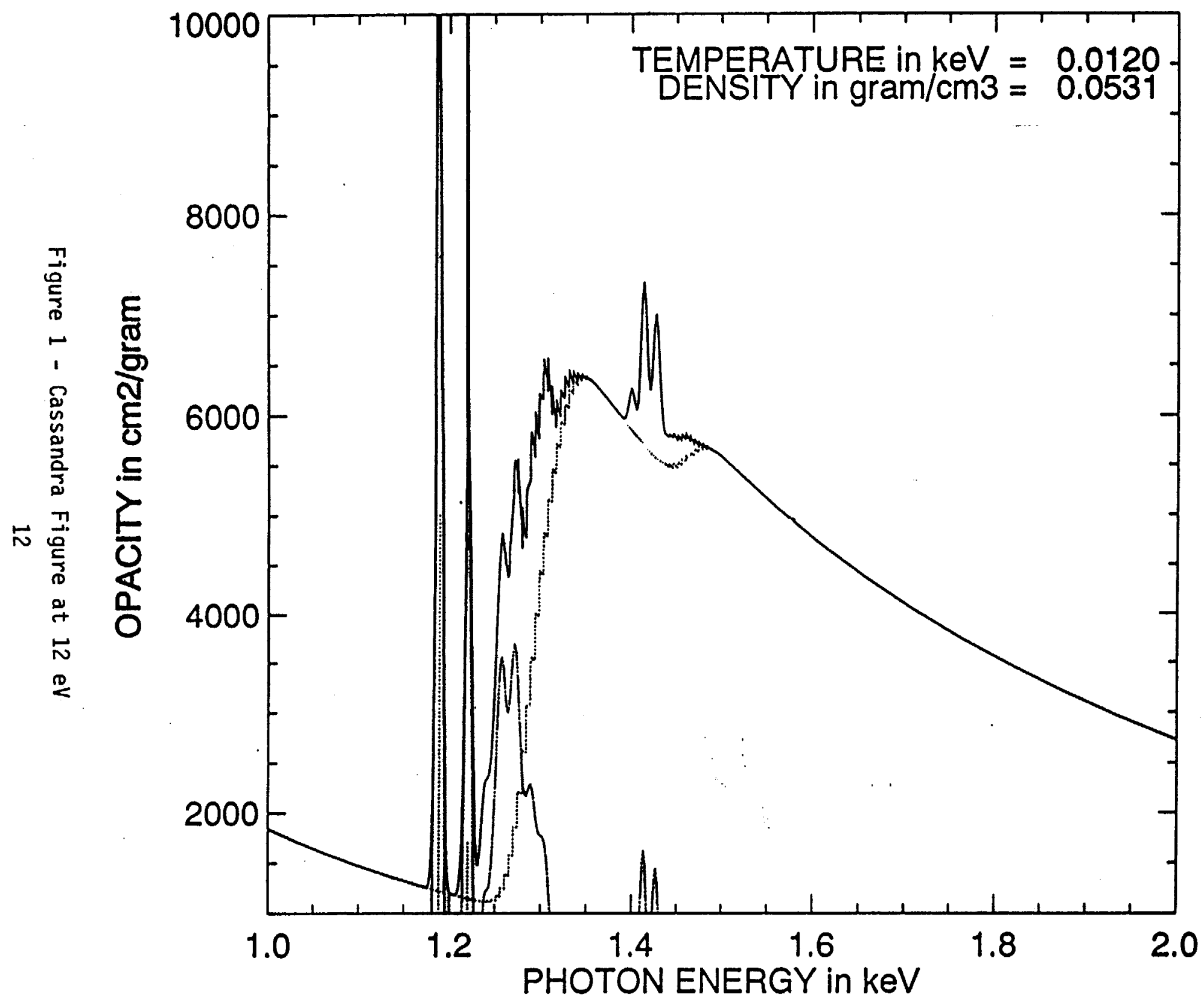




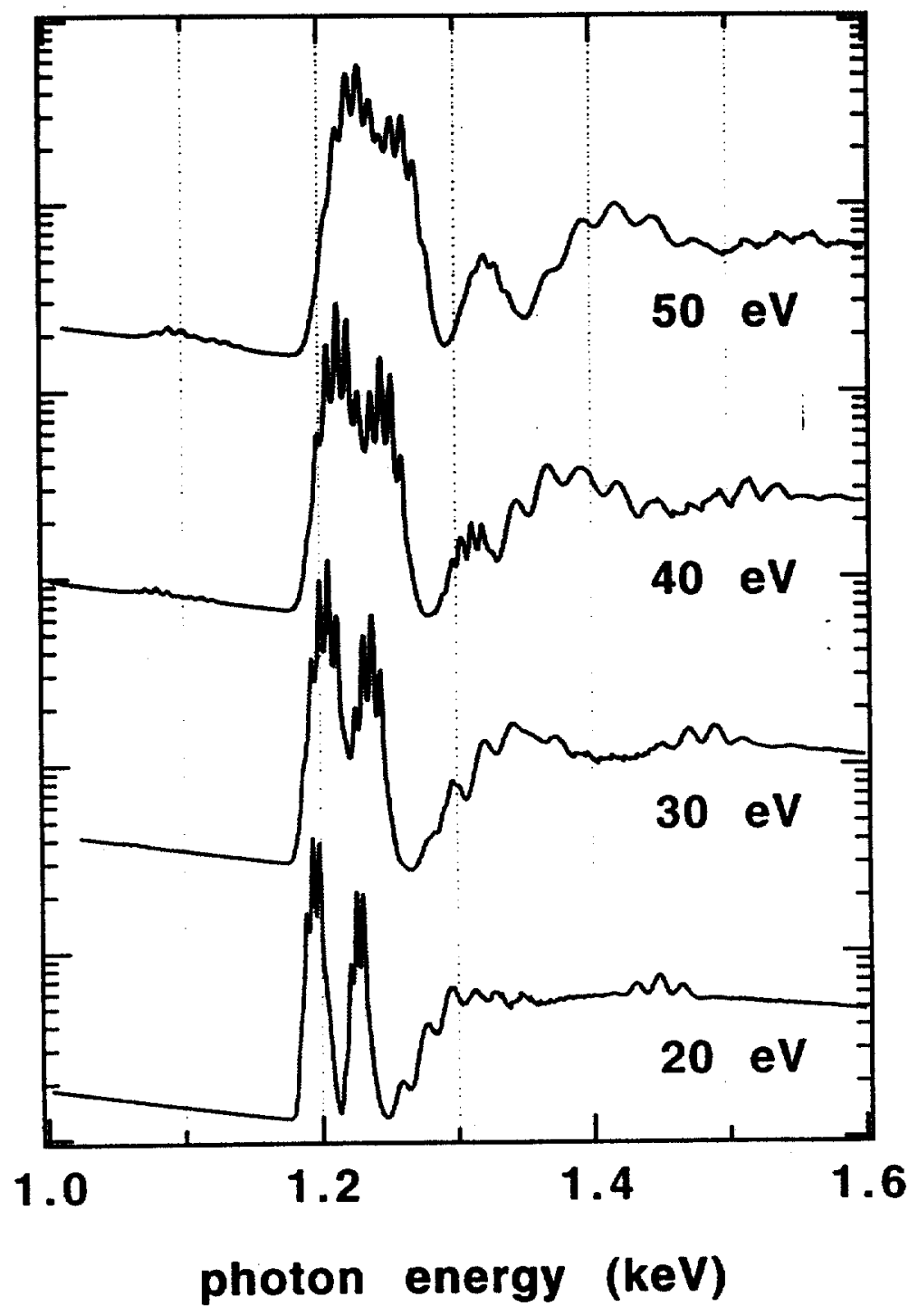

Figure 2 

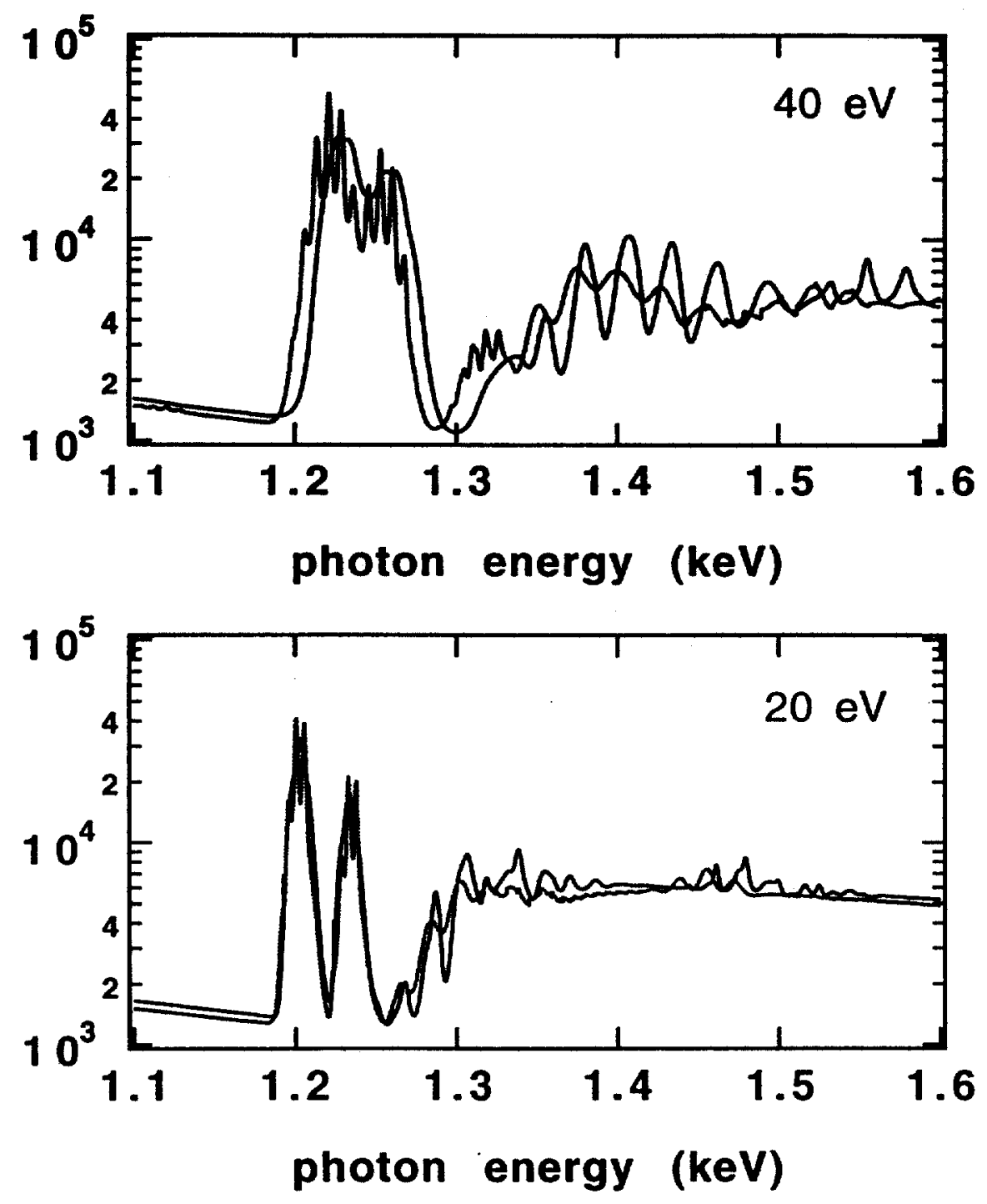

Figure 3. Cassandra vs STA at $40 \mathrm{eV}$ and $20 \mathrm{eV}$ 

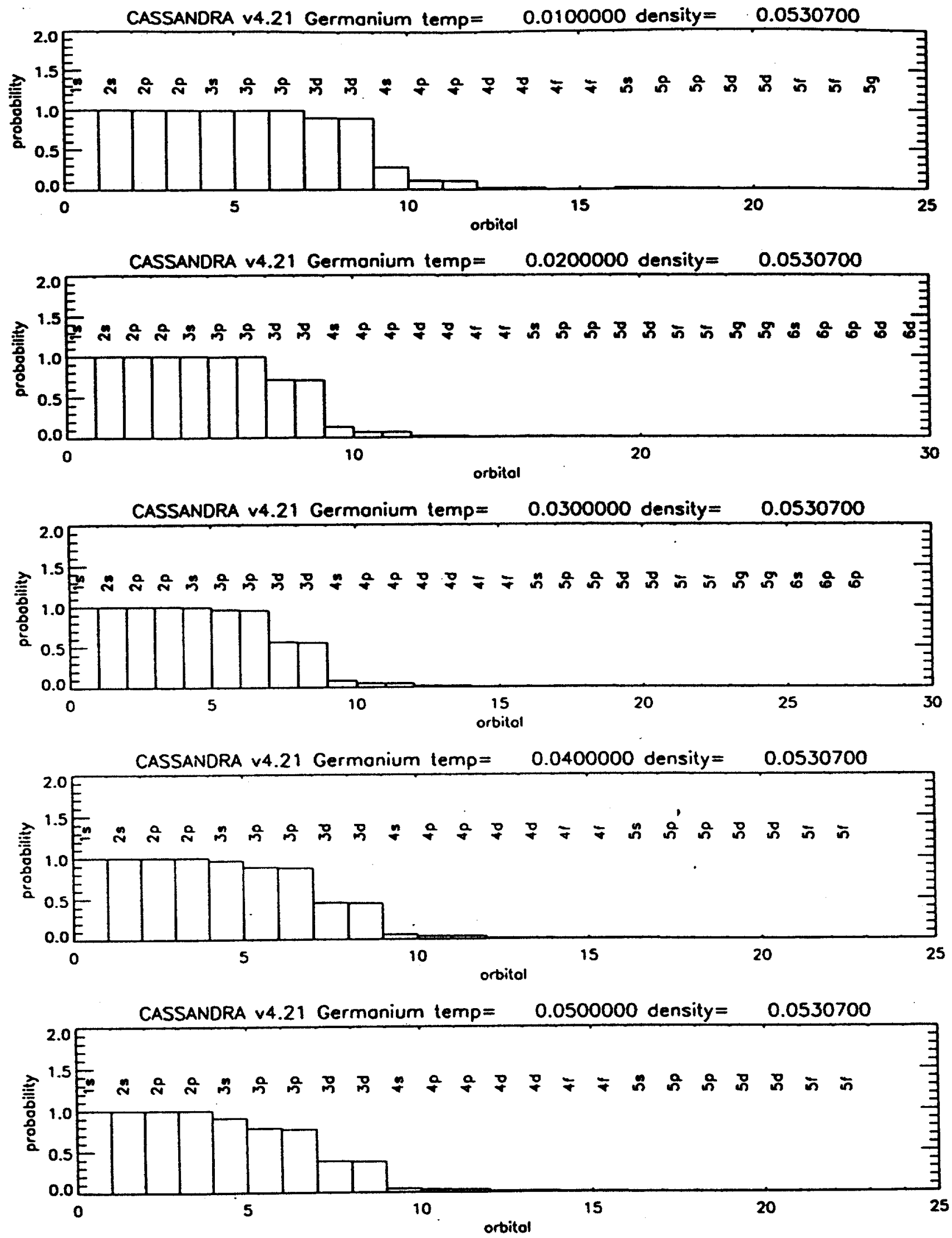

Figure 4. Ge populations in orbitals for $10,20,30,40$, and $50 \mathrm{eV}$ 


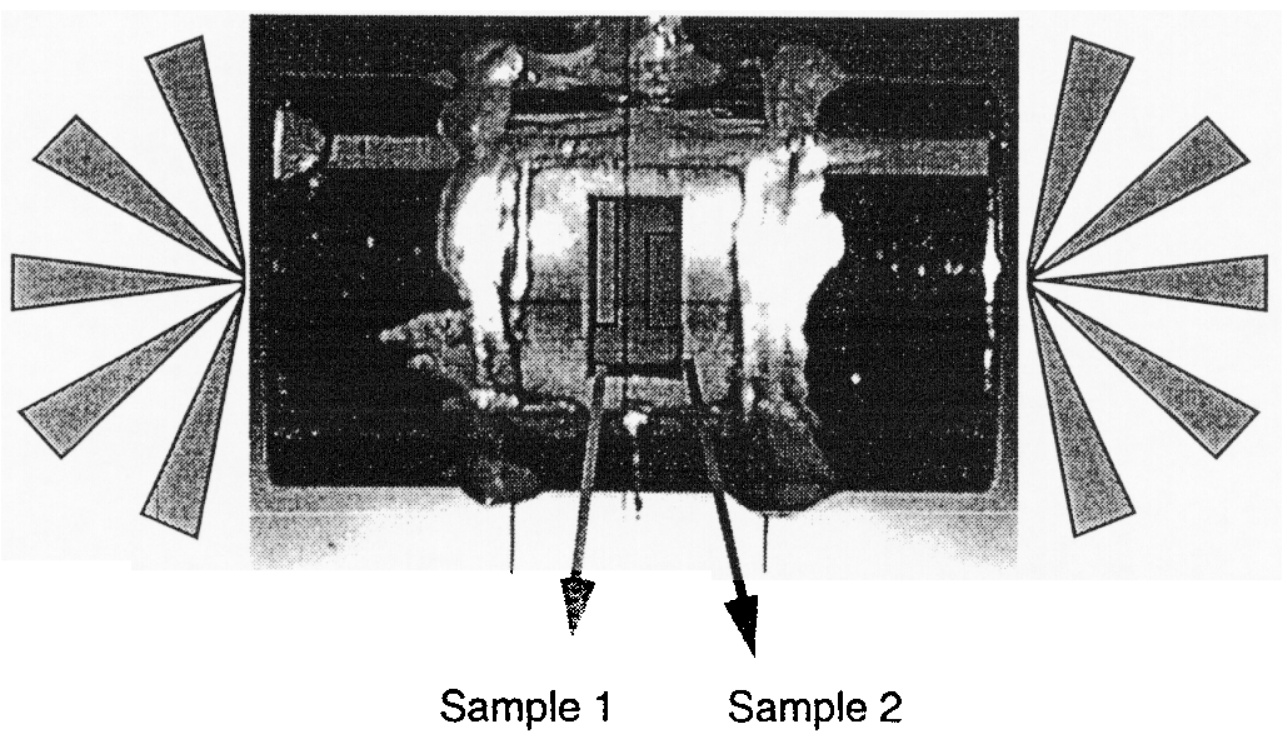

Figure 5. Experimental setup 


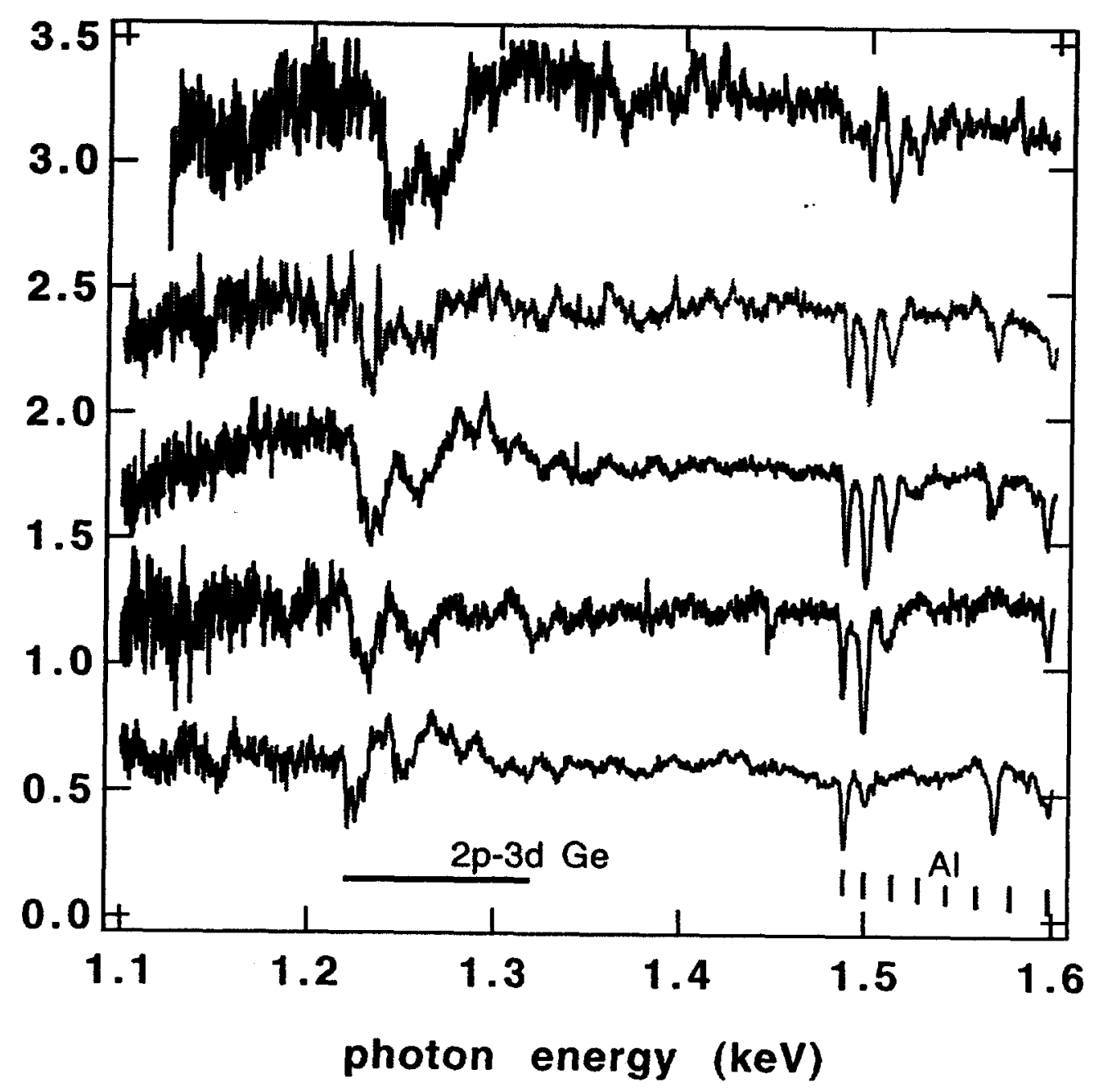

Figure 6. Experimental data 


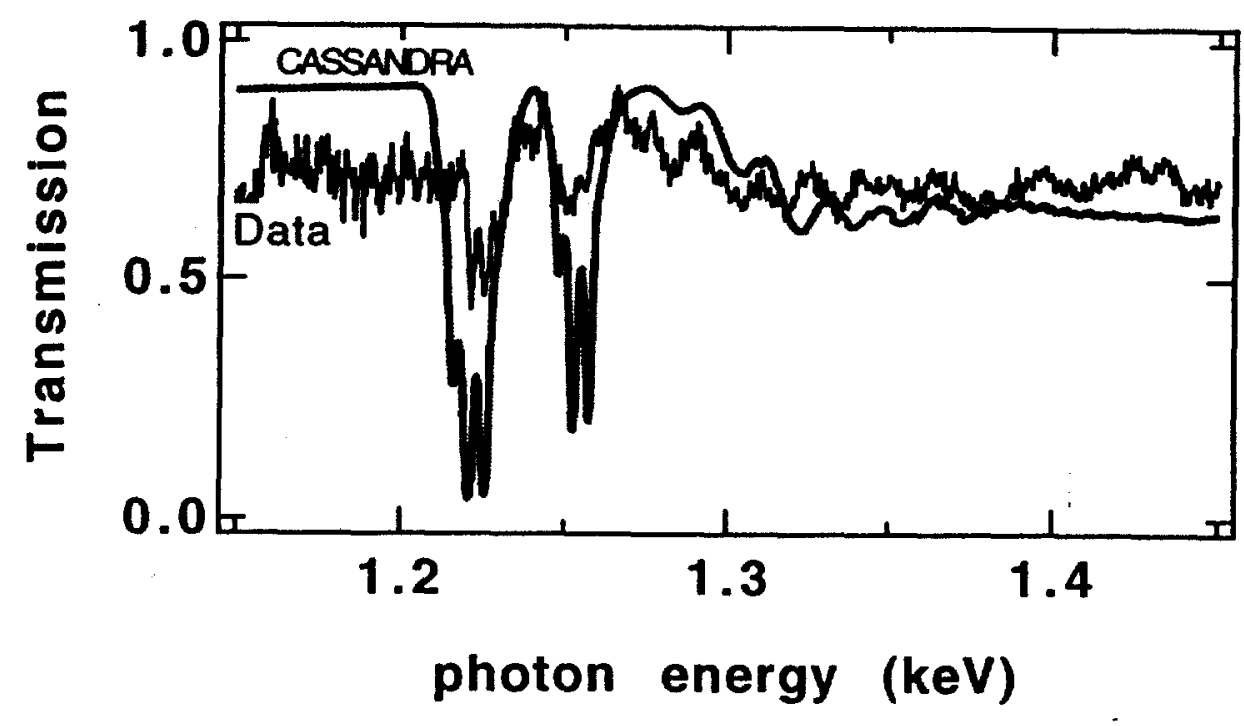

Figure 7. Comparison between experimental data and calculations for $20 \mathrm{eV}$ 


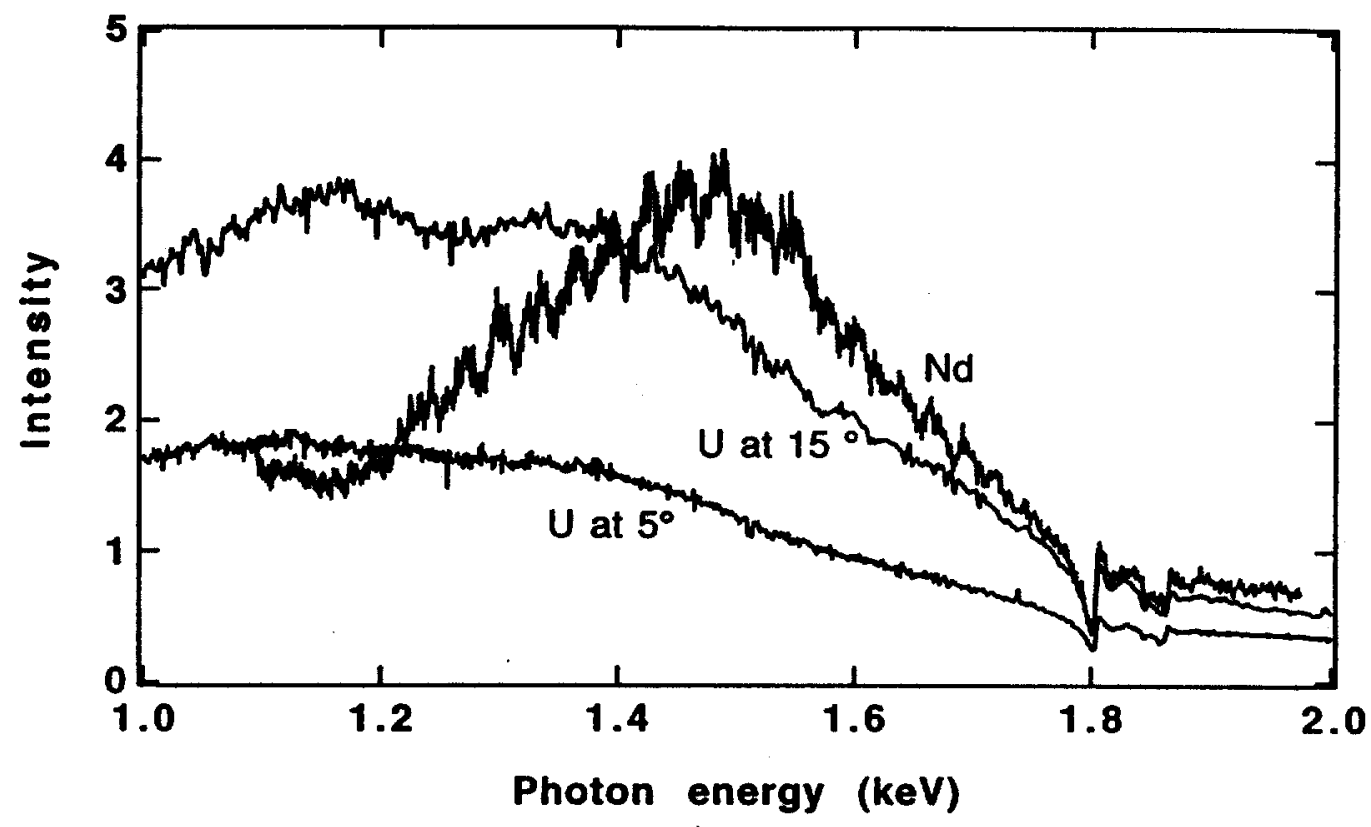

Figure 8. Nd vs U backlight spectra 


\section{REFERENCES}

${ }^{1}$ T. S. Perry, P. T. Springer, D. F. Fields, D. R. Bach, F. J. Serduke, C. A. Iglesias, F. J. Rogers, J. K. Nash, M. H. Chen, B. G. Wilson, W. H. Goldstein, B. Rozsynai, R. A. Ward, J. D. Kilkenny, R. Doyas, L. B. DaSilva, C. A. Back, R. Cauble, S. J. Davidson, J. M. Foster, C. C. Smith, A. Bar-Shalom, and R. W. Lee, Phys. Rev. E 54, 5617 (1996).

2L. DaSilva, B. J. MacGowan, D. R. Kania, B. A. Hammel, C. A. Back, E. Hsieh, R. Doyas, C. A. Iglesias, F. J. Rogers, and R. W. Lee, Phys. Rev. Lett. 69, 438 (1992).

3S. J. Davidson, J. M. Foster, C. C. Smith, and K. A. Warburton, and S. J. Rose, Appl Phys. Lett 52, 847 (1988).

${ }^{4}$ T. S. Perry, S. J. Davidson, F. J. D. Serduke, D. R. Bach, C. C. Smith, J. M. Foster, R. J. Doyas, R. A. Ward, C. A. Iglesias, F. J. Rogers, J. Abdallah, Jr., R. E. Stewart, J. D. Kilkenny, and R. W. Lee, Phys. Rev. Lett. 67, 3784 (1991).

5P. T. Springer, D. J. Fields, B. G. Wilson, J. K.Nash, W. H. Goldstein, C. A. Iglesias, F. J. Rogers, J. K. Swenson, M. H. Chen, A. Bar-Shalom, and R. E. Stewart, Phys. Rev. Lett. 69, 3735 (1992).

6B. J. B. Crowley, D. Rowley and D. K. K. Landeg, The CASSANDRA code, version 4 ( $\mathrm{AWE}, \mathrm{UK})$, private communication.

7 A. Bar-Shalom, J. Oreg, W. H. Goldstein, D. Shvarts, and A. Zigler Phys. Rev. A 40, 3183 (1989); A. Bar-Shalom, J. Oreg, and W. H. Goldstein. Phys. Rev. E 51, 4882 (1995). and references therein.

${ }^{8}$ T. S. Perry, K. S. Budil, R. Cauble, R. A. Ward, K. R. Bach, C. A. Iglesias, B. G. Wilson, J. K. Nash, C. C. Smith, J. M. Foster, S. J. Davidson, F. J. D. Serduke, J. D. Kilkenny, ad R. W. Lee, J. Quant. Spectrosc. Radiat. Transfer 54, 317 (1995). 
${ }^{9}$ F. J. Rogers, C. A. Iglesias, and B. G. Wilson, Ap. J. 397, 717 (1992).

${ }^{10}$ D. R. Kania, H. Kornblum, B. A. Hammel, J. Seely, C. Brown, U. Feldman, G. Glendinning, P. Young, E. Hsieh, M. Hennesian, L. DaSilva, B. J. MacGowan, D. S. Montgomery, C. A. Back, R. Doyas, J. Edwards, and R. W. Lee, Phys. Rev. A 46, 7853 (1992).

11D. A. Liberman, Phys.Rev. B 12, 4981 (1979)

12O. Gunnarsson and B I Lundqvist, Phys.Rev. B 13, 4274 (1976)

13B. J. B. Crowley, Phys.Rev. A 41, 2179 (1990)

14J. Stewart and K. Pyatt, Astrophys.J. 144, 1203 (1966)

15I. P. Grant, Adv.Phys. 19, 747 (1970)

16S. A. Moszkowski, Prog.Theor.Phys. 28, 1 (1962)

17 A. Goldberg and B. F. Rozsnyai, LLNL Report UCRL-95472 (1986)

18J. P. Cox and R. T. Giuli, Principles of Stellar Structure, vol.1 (Gordon and Breach, NY, 1968) chapter 2. 


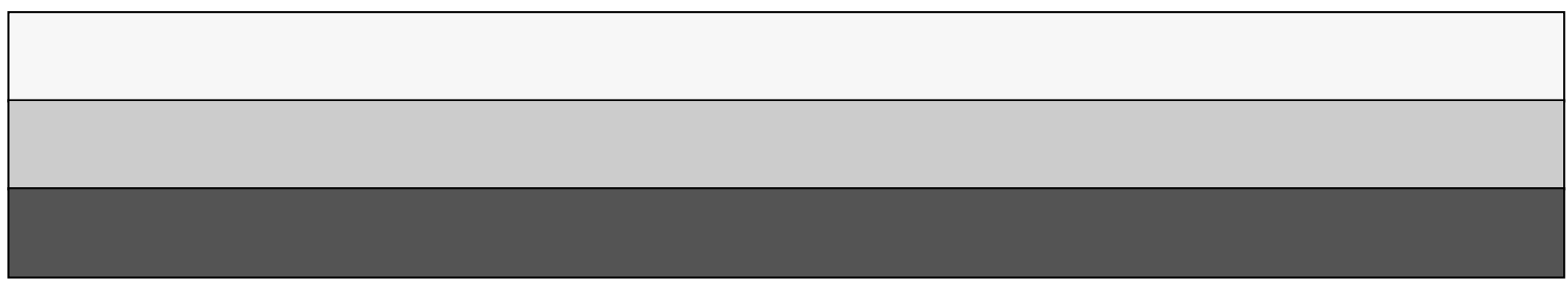

